\section{P389 DETECTION OF TREPONEMAL TP47 AND TP0548 GENES IN LESION SWABS FROM SYPHILIS PATIENTS}

${ }^{1} \mathrm{~F}$ Vasquez* ${ }^{*}{ }^{1,2} \mathrm{~S}$ Vargas, ${ }^{1} \mathrm{~J}$ Qquellon-Palacios, ${ }^{1} \mathrm{M}$ Eguiluz, ${ }^{3} \mathrm{G}$ Calvo, ${ }^{3} \mathrm{M}$ Reyes-Diaz, ${ }^{3,4} \mathrm{~K}$ Konda, ${ }^{3} \mathrm{C}$ Caceres, ${ }^{4} \mathrm{~J}$ Klausner. ${ }^{1}$ Sexual Health Laboratory, Center for Interdisciplinary Studies in Sexuality, AIDS and Society, Universidad Peruana Cayetano Heredia, Lima, Peru; ${ }^{2}$ School of Public Health and Administration, Universidad Peruana Cayetano Heredia, Lima, Peru; ${ }^{3}$ Center for Interdisciplinary Studies in Sexuality, AIDS and Society, Universidad Peruana Cayetano Heredia, Lima, Perú; ${ }^{4}$ Division of Infectious Diseases, David Geffen School of Medicine, University of California, Los Angeles, USA

10.1136/sextrans-2021-sti.424

Background Typical syphilis ulceration (chancre) can appear at the site of sexual contact during primary syphilis, and this lesion can be swabbed for detection of Treponema pallidum DNA. Two well-known gene targets for syphilis detection are tp47 and pol A with sensitivities between 60-70\%. The use of T. pallidum PCR in primary and secondary syphilis diagnosis and confirmation are recommended in European and CDC guidelines respectively. We evaluated tp47 and tp0548 as molecular screening targets; either target, if present, would indicate the presence of T. pallidum in the sample.

Methods Participants with syphilis clinical diagnosis were enrolled in a cohort study in Peru in 2019 and 2020. All participants were clinically examined to determine the presence of lesions. Lesion exudate was collected with a dacron swab and stored in a vial with $500 \mathrm{ul}$ of lysis buffer. T. pallidum DNA was extracted and tested using specific primers in conventional polymerase chain reaction (PCR) to amplify tp47 and tp0548 target genes.

Results Overall, 61/162 participants presented with lesions, and $27(43.5 \%)$ of them had T. pallidum DNA detected by either tp47 or tp0548 targets. The frequency of finding both targets in the same sample was $15 / 27$ (55.6\%). Only 1 sample was positive for tp47 but negative for tp0548, while 34 samples were negative for both T. pallidum targets. Additionally, only tp0548 was detected in 11 samples. We found tp47 in $16 / 61(26.2 \%)$ samples and tp0548 in 26/61 (42.6\%) samples. Adding the tp0548 screening target, increased the detection of T. pallidum by $69 \%$.

Conclusion tp0548 may help as a screening target to increase the detection of $\mathrm{T}$. pallidum in lesions. Thus both markers are necessary to increase sensitivity of detection T. pallidum DNA in lesions.

\section{P392 SEXUALLY TRANSMITTED INFECTIONS IN GOMBE, NORTH-EASTERN NIGERIA}

${ }^{1} \mathrm{M}$ Manga*, ${ }^{2} \mathrm{U}$ Hassan, ${ }^{1} \mathrm{~F}$ Halima, ${ }^{2} \mathrm{P}$ Bot, ${ }^{2} \mathrm{~B}$ Fatima, ${ }^{1} \mathrm{I}$ Mohammed, 'A Mohammed. ${ }^{1}$ Federal Teaching Hospital, Gombe, Nigeria; ${ }^{2}$ Gombe State University, Gombe, Nigeria

10.1136/sextrans-2021-sti.425

Background Sexually Transmitted Infections (STIs) have remained major sources of social and public health concern globally with worsening indices in developing countries and especially linked to the growing menace of antimicrobial resistance and poor infection prevention and control (IPC) measures. Diagnosis and treatment of STIs in low resource settings is still sub-optimal due to limited availability and or poor laboratory support with dearth of expertise in relevant disciplines. Many sexually transmitted pathogens are either fastidious or atypical thereby making their detection/identification more demanding. Establishment of special treatment clinics which are primarily concerned with managing STIs have been practiced with great successes across different parts of Nigeria. We for the first time present the pattern of STIs among patients attending the Special Treatment Clinic (STC) in Federal Teaching Hospital Gombe (FTHG) Nigeria.

Methods This was a hospital based cross sectional study in which sociodemographic and clinical/laboratory findings of 422 patients who presented at the STC of FTHG between 2015 and 2020 were analysed. The STC in FTHG is primarily concerned with management of STIs with in-built consulting/treatment room, side laboratory and records subunits.

Results Of the 422 patients, majority are married (73.7\%) and predominantly within the sexually active age group of 21-40 years $(80.7 \%)$. Female patients are more $(62.3 \%)$ than males and vaginal discharge is their most common (76.7\%) clinical presentation. Most common diagnoses among the patients are vulvovaginal/perineal candidiasis (24.9\%) and bacterial vaginosis (19.9). Others include genital ulcer disease; GUD (12.9\%), non-gonococcal urethritis; NGU (12.9\%), genital warts $(9.6 \%)$ and gonococcal urethritis/cervicitis (5.0\%).

Conclusion Vulvovaginal/perineal candidiasis and bacterial vaginosis are the most common diagnoses at the STC in Gombe Nigeria. The STC provides a convenient and effective avenue for the management of genital tract infections while hoping for expansion and improvement for better patient care and satisfaction.

\section{P393 FACTORS ASSOCIATED WITH INTEREST IN BACTERIAL SEXUALLY TRANSMITTED INFECTION VACCINES AMONG YOUNG HPV-VACCINATED CANADIAN WOMEN}

\begin{abstract}
${ }^{1,2} \mathrm{~A}$ de Waal ${ }^{*},{ }^{1,3} \mathrm{~S}$ Racey, ${ }^{1,3,4} \mathrm{R}$ Donken, ${ }^{1} \mathrm{~K}$ Plotnikoff, ${ }^{7} \mathrm{~S}$ Dobson, ${ }^{1,5} \mathrm{~L}$ Smith ${ }^{3,6} \mathrm{~T}$ Grennan, ${ }^{3,4} \mathrm{M}$ Sadarangani, ${ }^{1,3} \mathrm{G}$ Ogilvie. 'Women's Health Research Institute, $B C$ Women's Hospital and Health Service, Vancouver, Canada; ${ }^{2}$ McGill University, Faculty of Medicine, Montreal, Canada; ${ }^{3}$ University of British Columbia, Faculty of Medicine, Vancouver, Canada; ${ }^{4}$ Vaccine Evaluation Center, BC Children's Hospital Research Institute, Vancouver, Canada; ${ }^{5} B C$ Cancer, Vancouver, Canada; ${ }^{6} B C$ Centre for Disease Control, Vancouver, Canada; ${ }^{7}$ Sidra Medicine, Doha, Qatar
\end{abstract}

\subsection{6/sextrans-2021-sti.426}

Background Rates of bacterial sexually transmitted infections (STIs) are increasing globally, and these infections result in a large global burden of detrimental sexual, reproductive, and maternal-child health outcomes. Currently, the only prophylactic vaccines available against STIs are those for human papillomavirus (HPV) and hepatitis B. The objective of this study was to plan for future programme implementation by exploring acceptability, perceived barriers, and attitudes towards bacterial STI vaccines among young HPV-vaccinated Canadian women.

Methods A 20-item questionnaire was available from 06/ 2019-06/2020 to participants of the Canadian Quadrivalent HPV Vaccine Evaluation Study (QUEST). Multivariable logistic regression models assessed interest in chlamydia, syphilis, and gonorrhea vaccines using a priori clinically relevant variables and covariates significant at $\mathrm{p} \leq 0.05$ in bivariate analysis.

Results Surveys from 1092 respondents were analyzed, with $82 \%$ indicating interest in receiving one or more STI vaccines. $75 \%$ of respondents identified as white/European descent, with a median age of 19.6 years (range 17.5-23.0). In adjusted analyses, intent to engage in positive health behaviors was associated with vaccine interest for syphilis 
$(\mathrm{OR}=5.76,95 \% \mathrm{CI}[4.03-8.27])$, chlamydia $(\mathrm{OR}=5.27$, $95 \% \mathrm{CI}[3.66-7.63])$, and gonorrhea $(\mathrm{OR}=5.96,95 \% \mathrm{CI}$ [4.15-8.60]). Willingness to pay for an STI vaccine was also associated with vaccine interest for syphilis $(\mathrm{OR}=2.02$, 95\% CI [1.29-3.19]), chlamydia (OR $=2.41,95 \% \mathrm{CI}$ $[1.50-3.90])$, and gonorrhea $(\mathrm{OR}=2.29$, 95\% CI [1.443.63]). Ever having sexual intercourse and identifying as LGBTQ was significantly associated with vaccine interest in all adjusted models.

Conclusion Results indicate strong interest in receiving bacterial STI vaccines among young HPV-vaccinated Canadian women. Findings on acceptable cost, preferred age of administration, and anticipated health behaviours will allow for informed implementation of future bacterial STI vaccine programmes. Further exploration of STI vaccine acceptability among diverse populations in research and practice will inform effective vaccine rollout.

\section{P394 EVALUATION OF THE IN VITRO EFFICACY OF CEFIXIME, DALBAVANCIN, ISONIAZID, AND PYRAZINAMIDE AGAINST TREPONEMA PALLIDUM}

${ }^{1} \mathrm{~L}$ Tantalo*, ${ }^{2} \mathrm{M}$ Vall Mayans, ${ }^{2} \mathrm{M}$ Ubals Cazorla, ${ }^{3} \mathrm{C}$ Nieto, ${ }^{4,5} \mathrm{C}$ Pérez-Mañá, ${ }^{6,7} \mathrm{~L}$ Quintó, 2,8 $\mathrm{O}$ Mitja, ${ }^{1,9} \mathrm{~L}$ Giacani. ${ }^{1}$ Department of Medicine, Division of Allergy and Infectious Diseases, University of Washington, Seattle, USA; ${ }^{2}$ Fight Aids and Infectious Diseases Foundation, Hospital Germans Trias i Pujol, Barcelona, Spain; ${ }^{3}$ Laboratorio Reig Jofre, Barcelona, Spain; ${ }^{4}$ Universitari Germans Trias i Pujol, Institut de Recerca Germans Trias i Pujol, Badalona, Spain; ${ }^{5}$ Universitat Autònoma de Barcelona, Barcelona, Spain; ${ }^{6}$ ISGlobal, Hospital Clinic-Universitat de Barcelona, Barcelona, Spain; ${ }^{7}$ Manhiça Health Research Institute, Maputo, Mozambique; ${ }^{8}$ Lihir Medical Centre-International SOS, Lihir Island, Papua New Guinea; ${ }^{9}$ Department of Global Health, University of Washington, Seattle, USA

\subsection{6/sextrans-2021-sti.427}

Background The shortage in the supply of benzathine penicillin G (BPG) and other limitations associated with BPG use underscore the need for new therapeutic options for syphilis treatment. Until recently, Treponema pallidum (T. pallidum), the syphilis agent, could not be cultured in vitro, making the screening of antibiotics only possible in vivo, and hence difficult, time-consuming, and expensive. Because a cultivation system for this difficult pathogen is now available, we used an in vitro minimum inhibitory concentration (MIC) assay to screen antibiotics with the potential to provide new treatment options for syphilis.

Methods T. pallidum was cultured in vitro in the presence of multiple concentrations of cefixime, dalbavancin, isoniazid, and pyrazinamide in independent experiments. All these antibiotics have adequate pharmacokinetic and pharmacodynamic properties to treat syphilis based on previous data from humans studies in other infections. After seven days in culture, DNA was extracted from the culture wells and T. pallidum growth was compared to no-antibiotic culture wells using qPCR targeting the tp0574 gene.

Results Isoniazid and pyrazinamide, both antimycobacterials, had MICs of $>500 \mathrm{ng} / \mathrm{ml}$ and $>64 \mu \mathrm{g} / \mathrm{ml}$, respectively. For cefixime, a cephalosporin, and dalbavancin, a glycopeptide antibiotic, the experimental MICs were $<31.3 \mathrm{ng} / \mathrm{ml}$, and $<0.13 \mu \mathrm{g} / \mathrm{ml}$, respectively.

Conclusion Cefixime and dalbavancin appear to have marked microbicidal activity against T. pallidum with MICs that make them promising candidates for syphilis treatment in place of BPG. Isoniazid and pyrazinamide showed no significant treponemacidal activity in vitro at the concentrations tested.

\section{P398} COMMUNITY-INFORMED, ACADEMIC-PUBLIC HEALTH COLLABORATION TO DEVELOP AN ONLINE-LINKED MOBILE HIV/STI TESTING STRATEGY FOR GAY, BISEXUAL AND OTHER MSM IN MARYLAND

${ }^{1} \mathrm{E}$ Fields*, ${ }^{1} \mathrm{~N}$ Thornton, ${ }^{1} \mathrm{~S}$ Huettner, ${ }^{1} \mathrm{C}$ Schumacher, ${ }^{2} \mathrm{G}$ Barrow, ${ }^{2} \mathrm{~A}$ Greenbaum, ${ }^{1} \mathrm{~J}$ Jennings. ${ }^{1} J o h n s$ Hopkins School of Medicine, Baltimore, USA; ${ }^{2}$ Baltimore City Health Department, Baltimore, USA

10.1136/sextrans-2021-sti.428

Background Black MSM (BMSM) carry the greatest burden of new HIV diagnoses in the U.S. Ending the HIV epidemic (EHE) requires strategic and culturally-specific approaches to diagnosis, treatment and prevention. Increasingly, transmission is associated with online spaces where effective outreach strategies are limited. Through a community-informed, academicpublic health collaboration, we developed, implemented, and evaluated the feasibility and acceptability of an online-linked mobile van HIV/STI testing approach tailored to BMSM (SSP - Safe Spaces and Places) in one urban area.

Methods To assess feasibility of the Safe Spaces testing program, we compared populations recruited and testing outcomes to a public health mobile van HIV/STI testing program (PHD program) over 12 months. We also assessed participant acceptability of the SSP program through a survey assessing satisfaction and in-depth interviews.

Results Over 12-months, SSP tested 151 MSM (76\% Black; mean age $=34, S D=10.2)$. $7 \%(10 / 148)$ were new HIV diagnoses and $17 \%(26 / 150)$ were diagnosed with $>1$ bacterial STI. The PHD program tested 53\% (231) more MSM (70\% Black; mean age $=38, \mathrm{SD}=13.9)$, but yielded a significantly lower positivity rate; $0.5 \%(1 / 201, p=0.00)$ MSM were new HIV diagnoses and $0.04 \%(1 / 219, \mathrm{p}=0.00)$ were diagnosed with $>1$ bacterial STI. Of the $60 \%$ (90) of participants who completed a satisfaction survey, $83 \%$ (75) reported a positive experience and endorsed convenience, cost, and confidentiality as the most important reasons for choosing SSP. Twenty-five participants were interviewed and describing more privacy and less stigma concerns with SSP compared to other testing experiences.

Conclusions Our findings suggest that SSP was feasible, acceptable and may be more effective compared to standard PHD mobile testing in reaching high transmission risk BMSM. Future research initiatives will determine feasibility and effectiveness of linking at risk BMSM identified through this approach to PrEP or HIV care, key pillars of the EHE strategy.

\section{P399 EVALUATION OF COMMUNITY BASED HIV/STI TESTING PROGRAM TAILORED TO BLACK MSM IN A SOUTHEAST US CITY}

${ }^{1} E$ Fields*, 'G Aufderheide, ${ }^{2} B$ Silberzahn, ${ }^{1} S$ Huettner, ${ }^{1} \mathrm{~N}$ Thornton, ${ }^{1} \mathrm{~J}$ Jennings. ${ }^{1} J$ ohns Hopkins School of Medicine, Baltimore, USA; ${ }^{2}$ University of Texas at Austin, Austin, USA

\subsection{6/sextrans-2021-sti.429}

Background Black MSM (BMSM) are disproportionately burdened by HIV and STIs in the US. Structural barriers to HIV/ STI testing in clinical settings, including prior negative experiences, concerns about privacy/confidentiality and limited care access, compound disparities by contributing to delayed treatment and ongoing transmission. Testing modalities circumventing these barriers are needed for this priority population. 